\title{
Photoacoustic Imaging of Biological Tissues with High Cross-section Resolution
}

\author{
Xueding Wang, Yongjiang Pang, Minghua Xu, and Lihong V. Wang \\ Optical Imaging Laboratory, Biomedical Engineering Program, Texas A\&M University, TX 77843-3120, U.S.A. \\ URL: http://oilab.tamu.edu
}

\begin{abstract}
A modified back-projection approach deduced from an exact reconstruction solution was applied to photoacoustic tomography of differential absorption in biological tissues. Pulses from an Nd:YAG laser ( $8 \mathrm{~ns}$ FWHM at $1064 \mathrm{~nm}$ ) were employed to generate the distribution of acoustic sources in a sample. The acoustic signals were detected over a $2 \pi$ angle in an imaging plane that was perpendicular to the laser axis by a wide-band cylindrically focused ultrasonic transducer. Samples were made from slabs of pork fat in which several high absorption objects made from pig blood were buried. The reconstructed images agreed very well with the pictures of the samples. By employing our detection system and a reconstruction algorithm, a satisfying cross-section resolution that is better than $0.1 \mathrm{~mm}$ was achieved.

Keywords - Photoacoustic imaging, back-projection, cross-section resolution
\end{abstract}

\section{INTRODUCTION}

Photoacoustic imaging visualizes the high optical contrast, rather than the low acoustic contrast, between different soft biological tissues while at the same time retaining the satisfactory spatial resolution of pure ultrasound imaging. Thermal expansion of an absorbing structure in a tissue sample creates acoustic waves according to the thermoelastic mechanism, which can be detected by high sensitive piezoelectric devices outside the sample $[1,2]$. The absorption distribution in the sample can then be represented by applying a reconstruction algorithm.

In order to achieve high resolution in the $2 \mathrm{D}$ image of a cross section perpendicular to the laser axis, we arranged a wide-band ultrasound transducer around the laser axis to detect acoustic signals from the side of the sample. In order to expand the dynamic range of the signals from the imaging plane, the transducer had a cylindrical operating surface. The detected acoustic signals were processed through a modified back-projection approach deduced from an exact reconstruction solution.

\section{METHODS}

In this instance, we consider the imaging of an absorption distribution of a thin slice perpendicular to the laser axis. The detection of acoustic pressures over the $2 \pi$ angle in the imaging plane is sufficient to achieve a high cross-section resolution. For 2D tomography, the modified backprojection has the form:

$$
A(\mathbf{r}) \approx-\left.\frac{r_{0}^{2} C_{p}}{2 \pi I_{0} \beta c_{s}^{4}} \int_{\varphi_{0}} d \varphi_{0} \frac{1}{t} \frac{\partial p\left(\mathbf{r}_{0}, t\right)}{\partial t}\right|_{t=\frac{\left|r_{0}-r\right|}{c_{s}}},
$$

which is an integral over the angle $\varphi_{0} \in[0,2 \pi)$ within the imaging plane, where $A(\mathrm{r})$ describes the light absorption distribution at the position $\mathbf{r} ; r_{0}$ is the scanning radius; $I_{0}$ is a factor proportional to the incident radiation intensity; $c_{s}$ is the speed of the acoustic wave; $\beta$ and $C_{p}$ are the isobaric volume expansion coefficient and heat capacity (per unit mass); and $p\left(\mathrm{r}_{0}, t\right)$ is the pressure produced by the photoacoustic source. From Eq. (1), we see that the reconstruction of the absorption distribution can be fulfilled by back-projection of the quantity $-\left.\frac{1}{t} \frac{\partial p\left(r_{0}, t\right)}{\partial t}\right|_{t=\frac{\left|r_{0}-r\right|}{c_{s}}}$ instead of the acoustic pressure $p\left(\mathbf{r}_{0}, t\right)$.

With the impulse response of the detector $R(t)$ and the pulse duration of the laser $P(t)$ in the time domain, we have $T\left(\mathbf{r}_{0}, t\right)=p\left(\mathbf{r}_{0}, t\right) * R(t) * P(t)$, where $T\left(\mathbf{r}_{0}, t\right)$ is the piezoelectric signal detected by the transducer, and * represents convolution. Then, $\frac{\partial p\left(\mathbf{r}_{0}, t\right)}{\partial t}$ in Eq. (1) can be calculated by an inverse Fourier transformation,

$$
\frac{\partial p\left(\mathbf{r}_{0}, t\right)}{\partial t}=\frac{1}{2 \pi} \int_{-\infty}^{+\infty} \frac{-i \omega T\left(\mathbf{r}_{0}, \omega\right)}{P(\omega) R(\omega)} \exp (-i \omega t) d t
$$

The detailed deduction of our back-projection approach can be found in Ref. [3].

A schematic diagram of our experimental setup for photoacoustic tomography is shown in Fig. 1. A Nd:YAG laser provides $1064 \mathrm{~nm}$ laser pulses with a FWHM of $8 \mathrm{~ns}$ and a repetition rate of $10 \mathrm{~Hz}$. The laser pulse energy is approximately $400 \mathrm{~mJ}$. The laser is expanded to a $2.5 \mathrm{~cm}$ diameter beam when heating the sample surface from above along the laser axis; this provides an incident power density within the limit of safety for humans $\left(100 \mathrm{~mJ} / \mathrm{cm}^{2}\right)$. In our experiments, the area in a cross section of the sample that is imaged is defined by the size of the laser beam. The transducer was mounted on a rotation stage that was driven by a computer-controlled stepper motor. A low-noise pulse preamplifier (500 PR, Panametrics) amplified the acoustic signals received by the transducer and sent signals to an oscilloscope (TDS-640A, Tektronix).

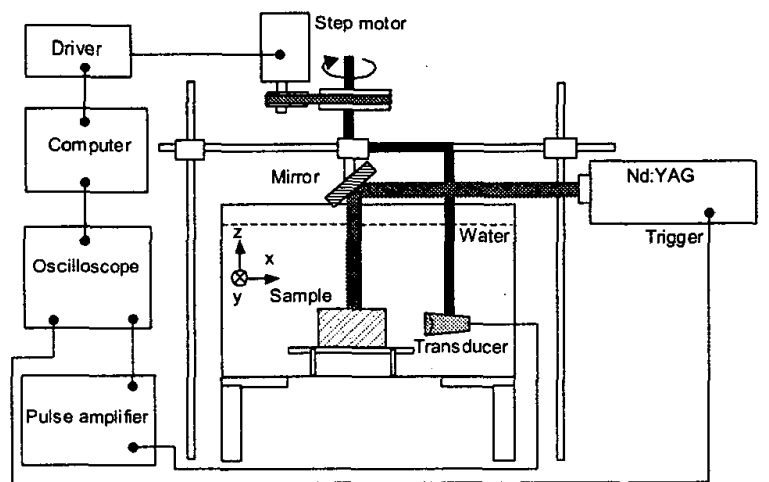

Fig. 1 The experimental setup. 


\section{RESULTS AND DISCUSSION}

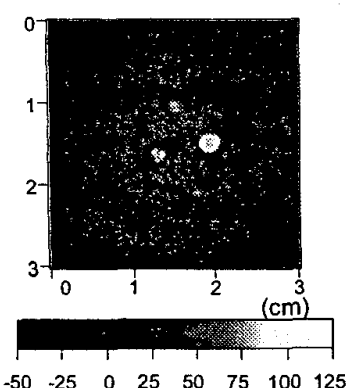

(a)

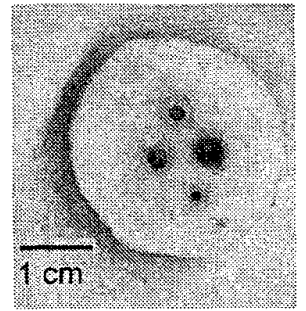

(b)
Fig. 2 (a) The photoacoustic image of four high absorption objects (pig blood) buried $0.4 \mathrm{~cm}$ deep in a slab of pig fat. (b) The picture of the cross section lying in the imaging plane of the sample.

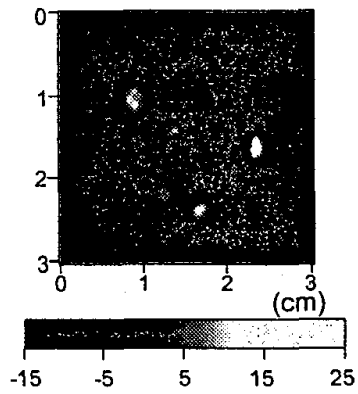

(a)

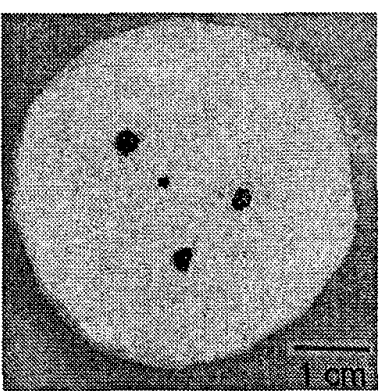

(b)
Fig. 3 (a) The photoacoustic image of four high absorption objects (pig blood) buried $1.5 \mathrm{~cm}$ deep in a slab of pig fat. (b) The picture of the cross section lying in the imaging plane of the sample.

The experiments were performed with slabs of pig fat tissues, in which several high absorption objects made from droplets of pig blood were buried. Image reconstruction utilized the 2D modified back-projection algorithm described in Eq. (1). The acoustic propagation speed $c_{s}$ was assumed to be $1.5 \mathrm{~mm} / \mu \mathrm{s}$. Fig. 2(a) shows the reconstructed image of four blood droplets buried $0.4 \mathrm{~cm}$ deep in a slab of fat tissue. The diameters of the four blood droplets are 3.2 $\mathrm{mm}, 2.1 \mathrm{~mm}, 1.5 \mathrm{~mm}$ and $0.8 \mathrm{~mm}$, respectively. The picture of the cross section of the sample that lies in the imaging plane is shown in Fig. 2(b) for comparison. We can see that the four absorption objects in the pig fat tissue with comparatively low absorbing coefficient are localized very well. The boundaries between the blood droplets and the fat are very clear. The imaged shape and size of each blood droplet shows a satisfying match with those of the sample.
Fig. 3(a) shows the reconstructed image of four blood droplets buried $1.5 \mathrm{~cm}$ deep in a slab of fat tissue. The sizes of the four blood droplets are around $3.0 \mathrm{~mm}, 2.2 \mathrm{~mm}, 2.2$ $\mathrm{mm}$ and $1.0 \mathrm{~mm}$, respectively. Compared with the picture of the cross section of the sample, the sizes and the localizations of the four blood droplets are imaged very well. In the reconstructed images in Figs. 2(a) and 3(a), we can see some intensity fluctuations around the absorbing objects, which come mainly from the photoacoustic signals generated in the background fat tissues.

\section{CONCLUSION}

Pulsed-laser induced photoacoustic imaging of differential absorption in biological tissues has been studied. A modified back-projection algorithm derived from an exact inverse solution was used to reconstruct the signals received by a wide-band cylindrical focused transducer that scanned along the side of the sample under detection. Reconstructed images of blood droplets buried in the pig fat tissue agreed very well with the pictures of samples. With our detection system as well as with the reconstruction algorithm, the cross-section resolution is better than $0.1 \mathrm{~mm}$.

Our photoacoustic detection system with the modified back-projection reconstruction algorithm is proved to be an effective method for biological tissue cross-section imaging with high contrast and high spatial resolution. Imaging results of some organs of small animals, such as the mouse kidney, etc., will also be presented during the conference.

\section{ACKNOWLEDGMENT}

This project was sponsored in part by the U.S. Army Medical Research and Material Command Grant No. DAMD 17-00-1-0455, the National Institutes of Health Grant No. R01 CA71980, the National Science Foundation Grant No. BES-9734491, and Texas Higher Education Coordinating Board Grant No. ARP 000512-0063-2001.

\section{REFERENCES}

[1] A. A. Oraevsky, V. A. Andreev, A. A. Karabutov, D. R. Fleming, Z. Gatalica, H. Singh, and R. O. Esenaliev, "Laser Opto-Acoustic Imaging of the breast: Detection of Cancer Angiogenesis", Proc. SPIE, vol. 3597, pp. 352-363, 1999.

[2] C. G. A. Hoelen, F. F. M. de Mul, R. Pongers, and A. Dekker, "Threedimensional photoacoustic imaging of blood vessels in tissue", Opt. Lett., vol. 23, pp. 648-650, 1998.

[3] M. Xu and L. V. Wang, "Pulsed-microwave-induced thermoacoustic tomography of biological tissues: Reconstruction theory and experiments", IEEE T. Med. Imaging, in press. 